\title{
Dans la continuité
}

\section{L'assemblée générale 2002 de la SMF}

\section{L} 'assemblée générale 2002 de la Société météorologique de France (SMF) s'est tenue à Paris, à la Maison de la chimie, le lundi 29 avril dernier à 15 heures. Michel Petit, président de la SMF, a ouvert la réunion devant une assistance quelque peu clairsemée.

Le secrétaire général, René Morin, a ensuite présenté le rapport moral sur l'exercice 2001, en énumérant les nombreuses actions menées par la SMF au cours de l'année passée. Parmi les plus marquantes, retenons simplement ici :

- La préparation de la célébration du $150^{\mathrm{e}}$ anniversaire de la SMF, à laquelle a dû s'atteler Michel Petit dès sa nomination à la présidence de la société. La SMF a décidé d'organiser en 2002 une manifestation intitulée «L'air de la ville », destinée au grand public et portant sur le thème de la pollution urbaine, et cela en partenariat avec Météo-France, la Ville de Paris, Airparif et plusieurs autres organismes. À cette fin, la SMF a mis en place un comité de programme, présidé par Jean-Pierre Javelle, et un comité d'organisation, présidé par Morgane Daudier. La manifestation comportera trois volets (pour plus de détails, voir la rubrique «Annonces ») :

- une exposition à l'hôtel de ville de Paris, qui présentera au public, du 13

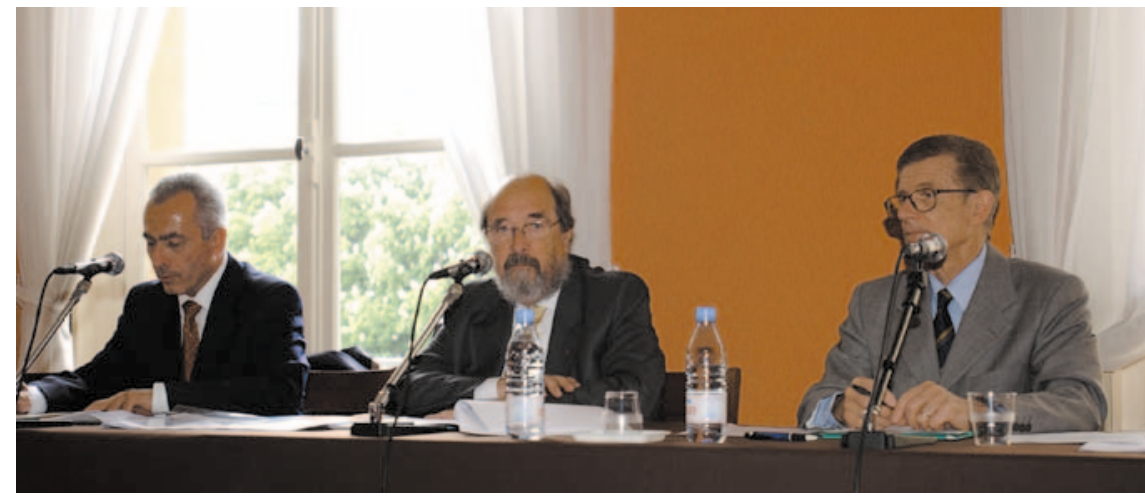

L'ouverture de l'assemblée générale 2002. De gauche à droite : Christian Lafayne, trésorier de la SMF, Michel Petit, président de la SMF, et René Morin, secrétaire général de la SMF. (Photos Météo-France, Pascal Taburet)

au 27 novembre 2002, des panneaux pédagogiques sur la pollution atmosphérique, des instruments de mesure et des informations en temps réel sur la pollution de l'air en région parisienne ; - un cycle de conférences autour de la pollution atmosphérique, pendant la même période et au même endroit ;

- un concours photo sur les phénomènes météorologiques et la pollution en milieu urbain.

- La bonne santé de la section MidiPyrénées de la SMF, dont le cycle de conférences grand public s'est poursuivi en 2001 avec un succès certain.

- La réussite de la $3^{\text {e }}$ Rencontre Météo Jeunes, qui a fait venir pratiquement une centaine de participants les 19 et 20 mai 2001 à Toulouse.

Le dépouillement du vote pour le renouvellement du conseil, une opération très sérieuse ! De gauche à droite, Georges Dhonneur, Nathalie Conchon et Morgane Daudier.

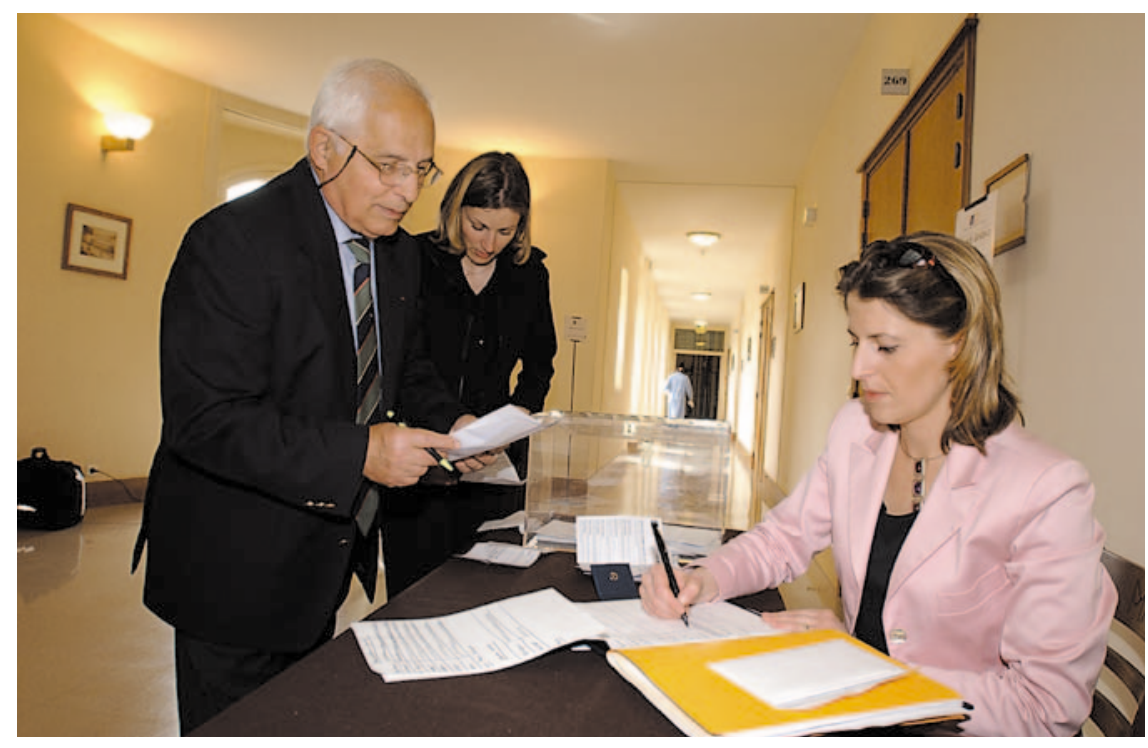

Michel Petit a alors repris la parole et informé l'assemblée de la décision du conseil de la SMF de demander au trésorier de revoir la présentation du rapport financier sur l'exercice 2001. Bien qu'il n'y ait pas de problème de fond sur cet exercice financier, la façon dont les comptes sont présentés n'a pas permis de les soumettre à l'assemblée générale. Le rapport financier 2001 sera donc présenté ultérieurement, soit lors d'une nouvelle assemblée générale, soit par correspondance. En outre, Michel Petit a souligné qu'en raison d'un retard dans le versement de la subvention du ministère des Affaires étrangères, subvention destinée à aider les stagiaires étrangers à payer leurs frais de séjour en France, l'apparente abondance des fonds de la SMF avait disparu. Le président a enfin fait part d'un léger déficit du budget annuel de la SMF et de son intention de trouver les moyens de ramener le budget à l'équilibre.

Le trésorier, Christian Lafayne, a ensuite présenté à l'assemblée le budget prévisionnel 2002 de la SMF. Retenons simplement la dépense exceptionnelle de 30000 euros prévue pour financer la célébration du $150^{\circ}$ anniversaire. Christian Lafayne a également soumis au vote de l'assemblée les nouveaux tarifs d'adhésion à la SMF et d'abonnement à la revue $L a$ Météorologie, tarifs qui entreront en vigueur au $1^{\text {er }}$ janvier 2003.

C'était alors le moment de la proclamation des résultats du vote pour le renouvellement du conseil de la 


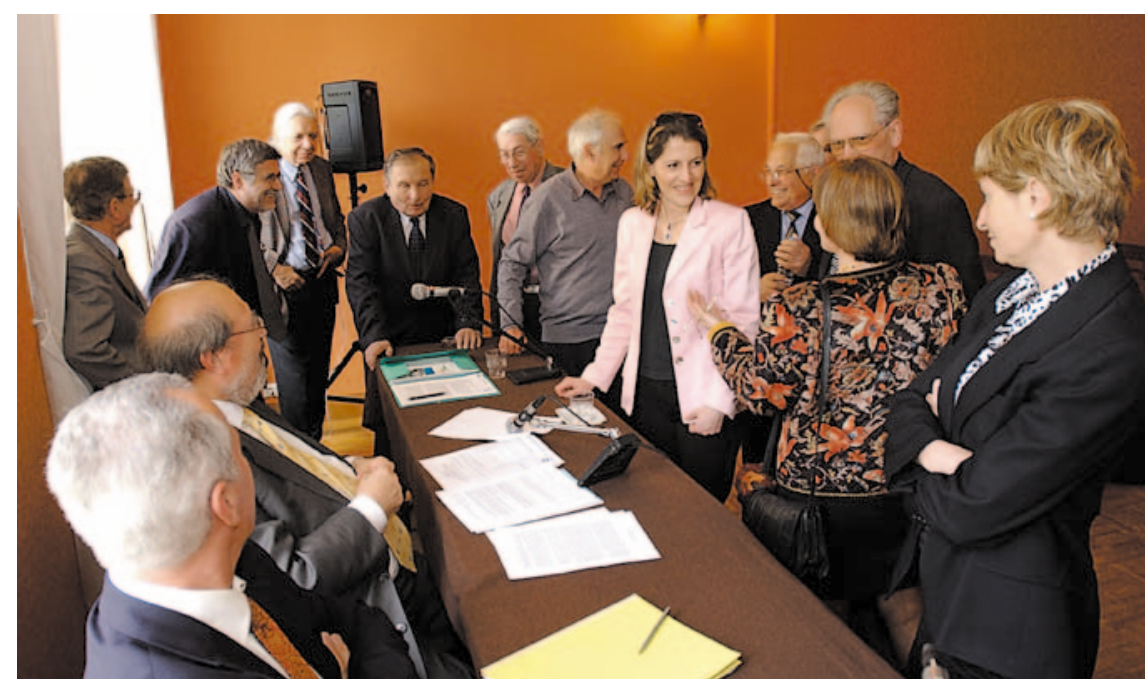

Le nouveau conseil de la SMF vient de désigner son bureau.

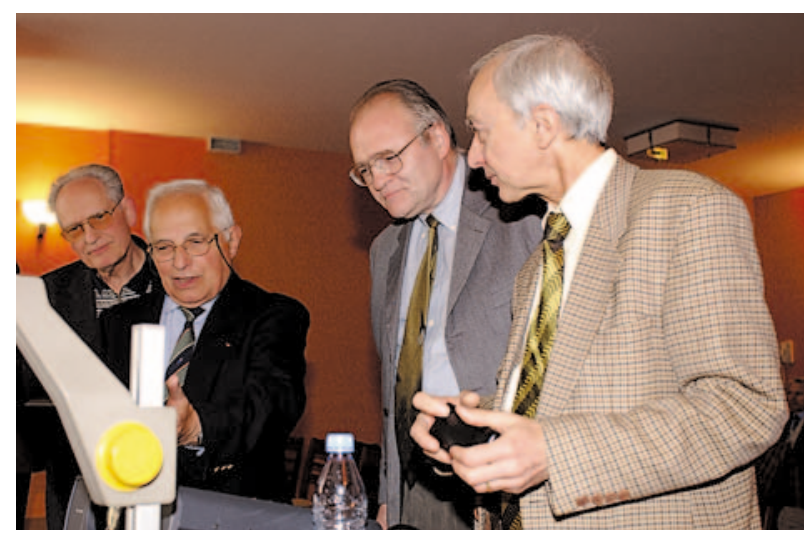

Commentaires passionnés devant le rétroprojecteur informatique. De gauche à droite : Guy Blanchet, Georges Dhonneur, Jean-François Geleyn et Claude Pastre.

SMF. Seul changement pour 20022003 : Véronique Bourgeois (MétéoFrance) a été élue en remplacement de Jacques Darchen, qui n'avait pas souhaité se représenter. Le conseil s'est alors brièvement réuni pour désigner son bureau. On trouvera fiabilité toujours accrue des prévisions du temps, il a mis l'accent sur les trois prochains rendez-vous suivants :

- la $4^{\mathrm{e}}$ Rencontre Météo Jeunes à Toulouse ;

- la célébration du $150^{\mathrm{e}}$ anniversaire de la SMF ;

Jean-François Geleyn s'apprête à nous montrer que le calcul numérique n'est pas l'ennemi des prévisionnistes.

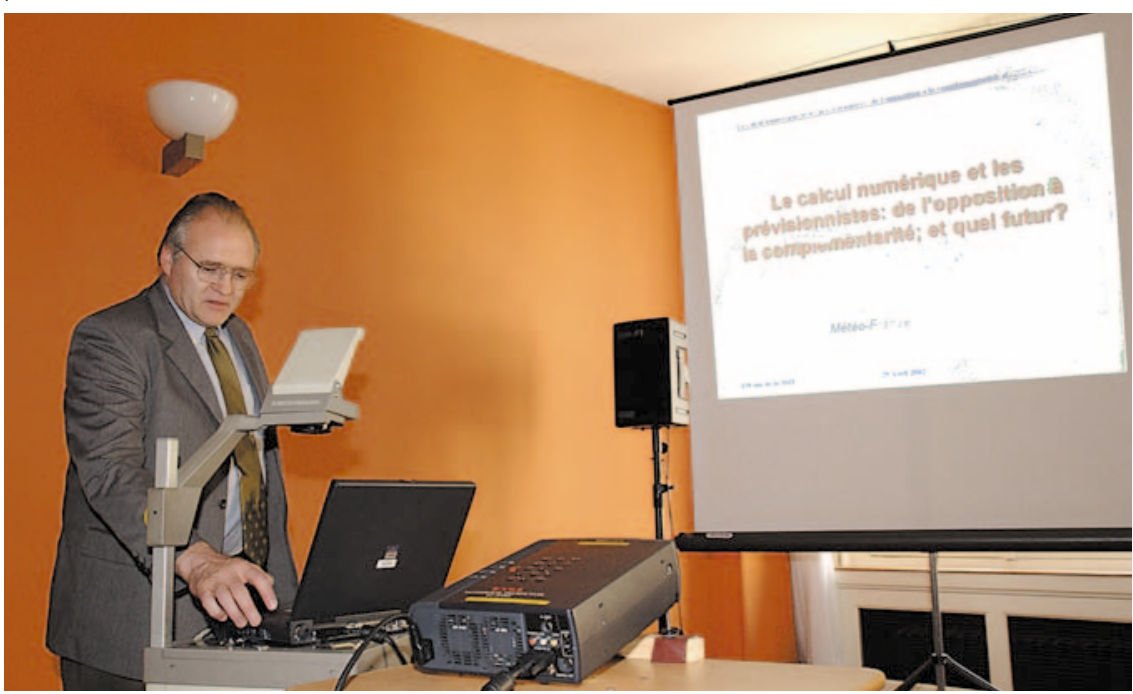

- l'organisation d'un nouveau colloque «Météorologie et savoir », faisant suite à celui de 1997 à Arc-et-Senans, probablement en 2004.

Le moment était venu pour l'assistance, renforcée par l'arrivée de plusieurs personnes de Météo-France, d'écouter la conférence présentée par Jean-François Geleyn, responsable de la recherche en prévision numérique au Centre national de recherches météorologiques (CNRM) de Météo-France. La conférence était intitulée « Le calcul numérique et les prévisionnistes : de l'opposition à la complémentarité. Et quel futur?».

En cinquante minutes environ, JeanFrançois Geleyn nous a montré que l'ancienne opposition entre les prévisionnistes et la prévision numérique du temps avait été surmontée. À Météo-France en particulier, la complémentarité de l'expertise humaine et du calcul automatique est désormais au cœur des grands programmes

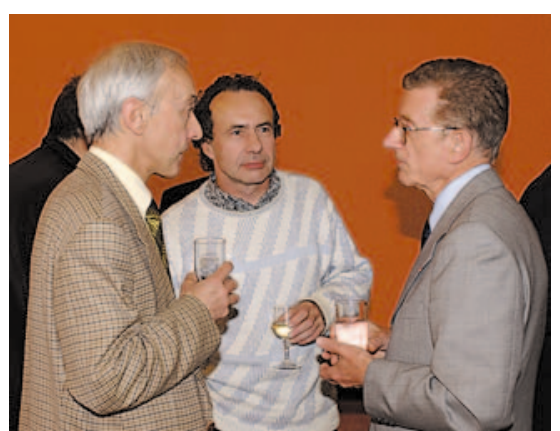

Pendant le cocktail de fin d'après-midi. De gauche à droite : Claude Pastre, Jacques Siméon et René Morin.

dans le domaine de la prévision, à savoir : les nouveaux outils graphiques Anasyg et Presyg, la production Symposium de bases de données prévues, le terminal interactif Synergie pour le prévisionniste et les modèles de prévision numérique existants (Arpège et Aladin) et futurs (Arome). Félicitons Jean-François Geleyn d'avoir su mettre à la portée de son auditoire un sujet fort difficile, que ce soit par ses arrière-plans scientifiques ou par ses implications techniques et organisationnelles.

L'après-midi s'est achevée autour du sympathique cocktail offert par la Société météorologique de France.

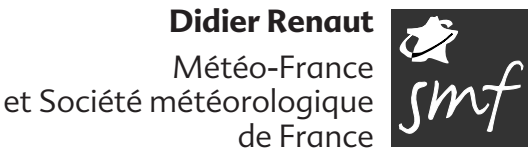




\title{
Conseil d'administration et bureau de la SMF
}

\author{
(après l'assemblée générale 2002)
}

Exercice 2002-2003

\begin{tabular}{|c|c|c|}
\hline \multicolumn{3}{|l|}{ Bureau } \\
\hline Président & Michel Petit & $\begin{array}{l}\text { Ingénieur général des télécommunications } \\
\text { Ancien directeur général adjoint chargé de la recherche à l'École polytechnique } \\
\text { Membre du Bureau du Groupe intergouvernemental d'experts sur l'évolution } \\
\text { du climat (Giec) }\end{array}$ \\
\hline Président honoraire et secrétaire général & René Morin & Ancien directeur délégué à la Société européenne de propulsion (SEP) \\
\hline \multirow[t]{3}{*}{ Vice-présidents } & Georges Dhonneur & Ingénieur général de la météorologie (C.H.) \\
\hline & Pierre de Félice & $\begin{array}{l}\text { Professeur à Paris XII (université de Créteil) } \\
\text { Laboratoire de météorologie dynamique de l'École polytechnique }\end{array}$ \\
\hline & Marc Gillet & $\begin{array}{l}\text { Ingénieur général de la météorologie } \\
\text { Directeur de l'Observatoire national sur les effets du réchauffement climatique } \\
\text { en France }\end{array}$ \\
\hline Trésorier & Christian Lafayne & $\begin{array}{l}\text { Ingénieur divisionnaire de la météorologie } \\
\text { Météo-France, département " Défense " }\end{array}$ \\
\hline Conseiller & André Lebeau & $\begin{array}{l}\text { Ancien professeur au Conservatoire national des arts et métiers (Cnam) } \\
\text { Ancien directeur général de Météo-France } \\
\text { Ancien président du Centre national d'études spatiales (Cnes) }\end{array}$ \\
\hline Conseillère & Françoise Désalmand & Enseignant-chercheur à l'université Paris VI \\
\hline Conseiller technique & Jean Galzi & Ingénieur général de la météorologie (C.H.) \\
\hline Responsable de la section Haute-Garonne & Olivier Moch & $\begin{array}{l}\text { Ingénieur général de la météorologie } \\
\text { Météo-France, directeur général adjoint }\end{array}$ \\
\hline Responsable des conférences grand public & Claude Pastre & $\begin{array}{l}\text { Ingénieur général de la météorologie } \\
\text { Coordinateur du réseau Eumetnet }\end{array}$ \\
\hline $\begin{array}{l}\text { Chargée de la communication } \\
\text { et des affaires générales }\end{array}$ & Morgane Daudier & Administrateur de la SMF \\
\hline
\end{tabular}

Autres membres du conseil d'administration

\begin{tabular}{|c|c|c|}
\hline Membre de droit & Jean-Pierre Beysson & $\begin{array}{l}\text { Président-directeur général de Météo-France } \\
\text { Conseiller maître à la Cour des comptes }\end{array}$ \\
\hline \multirow[t]{12}{*}{ Membres } & Jean-Claude André & $\begin{array}{l}\text { Ingénieur général de la météorologie } \\
\text { Directeur du Cerfacs }\end{array}$ \\
\hline & Guy Blanchet & Directeur honoraire du Centre de climatologie (université de Lyon I) \\
\hline & Véronique Bourgeois & $\begin{array}{l}\text { Technicienne supérieure de la météorologie } \\
\text { Météo-France, Direction des systèmes d'information }\end{array}$ \\
\hline & Jean Bourgoin & $\begin{array}{l}\text { Ingénieur général de l'armement (C.H.) } \\
\text { Ancien directeur de l'Epshom }\end{array}$ \\
\hline & Patrick Brochet & Ingénieur général de la météorologie (C.H.) \\
\hline & Olivier Carel & $\begin{array}{l}\text { Ingénieur général de l'aviation civile } \\
\text { Conseiller scientifique du directeur de la DNA }\end{array}$ \\
\hline & Jean Cassanet & Professeur, expert Espace-météorologie au ministère de la Recherche \\
\hline & Bertrand Imbert & Ingénieur hydrographe (C.H.) \\
\hline & Gérard Lambert & $\begin{array}{l}\text { Directeur de recherche au CNRS (C.H.) } \\
\text { Ancien responsable du Centre des faibles radioactivités }\end{array}$ \\
\hline & Hervé Le Men & $\begin{array}{l}\text { Ingénieur en chef géographe } \\
\text { Responsable de la recherche à l'IGN }\end{array}$ \\
\hline & Didier Renaut & $\begin{array}{l}\text { Ingénieur en chef de la météorologie } \\
\text { Météo-France, rédacteur en chef de La Météorologie }\end{array}$ \\
\hline & Frank Roux & $\begin{array}{l}\text { Directeur de recherche au CNRS } \\
\text { Laboratoire d'aérologie, université Paul-Sabatier }\end{array}$ \\
\hline
\end{tabular}

